ANNALES

POLONICI MATHEMATICI

$83.3(2004)$

\title{
Analytic solutions for polynomial-like iterative equations with variable coefficients
}

\author{
by BING Xu (Chengdu)
}

\begin{abstract}
Analytic solutions of polynomial-like iterative functional equations with variable coefficients are discussed in the complex field $\mathbb{C}$ by reducing to an auxiliary equation and by applying known results for systems of nonlinear functional equations of finite orders.
\end{abstract}

1. Introduction. An important class of functional equations with iterates of unknown functions $([2,3,10])$ is the polynomial-like iterative equations

$$
\lambda_{1} f(x)+\lambda_{2} f^{2}(x)+\cdots+\lambda_{n} f^{n}(x)=F(x),
$$

where the function $F$ and the constants $\lambda_{k}(k=1, \ldots, n)$ are given, $f$ is the unknown function and $f^{k}$ denotes the $k$ th iterate of $f$ (i.e., $f^{0}(x)=x$, $\left.f^{k}(x)=f\left(f^{k-1}(x)\right), k=1,2, \ldots\right)$. For real solutions, results on continuity, differentiability and symmetries are given for example in $[4,11-14]$. On the other hand, for complex solutions, analyticity is also discussed in $[6,7]$.

It is also interesting to study equation (1.1) with variable coefficients, i.e., when all $\lambda_{k}$ 's are functions of $x$, denoted by $\lambda_{k}(x)(k=1, \ldots, n)$. It is well known that linear ordinary differential equations with variable coefficients are much more complicated than those with constant coefficients. With such motivation, existence of continuous solutions for equation (1.1) with variable coefficients was investigated in [15] and its differentiable solutions were further discussed in [8]. However, no result on analytic solutions has been given.

In this paper, under the basic assumption that

(H) $\quad \lambda_{k}(k=1, \ldots, n)$ and $F$ are analytic complex-valued functions in a neighborhood of the origin in the complex field $\mathbb{C}$ and $F(0)=0$,

2000 Mathematics Subject Classification: 39B12, 37E05.

Key words and phrases: iterative equation, variable coefficients, analytic solution, polynomial.

Supported by NSFC (China) Grant and China MOE Research Grants. 
we study local analytic solutions of the functional equation

$$
\lambda_{1}(z) f(z)+\lambda_{2}(z) f^{2}(z)+\cdots+\lambda_{n}(z) f^{n}(z)=F(z)
$$

where $n \geq 2$ is a fixed positive integer and $f$ is the unknown function. Our main result is the following:

THEOREM. Suppose that hypothesis $(\mathrm{H})$ holds and that $\alpha_{1}, \alpha_{2}$ are zeros of the polynomial $P(z):=\sum_{k=1}^{n} \lambda_{k}(0) z^{k}-F^{\prime}(0)$ in $\mathbb{C}$ with the smallest and largest absolute value respectively. If either $(\mathrm{A})\left|\alpha_{1}\right|<1$ and $F^{\prime}(0) \neq 0$, or (B) $\left|\alpha_{2}\right|>1$ and $\lambda_{n}(0) \neq 0$, then equation (1.2) has a local analytic solution $f$ such that $f(0)=0$ and $f^{\prime}(0)=\alpha$, where $\alpha=\alpha_{1}$ in case $(\mathrm{A})$ and $\alpha=\alpha_{2}$ in case $(\mathrm{B})$.

Such an analytic solution will be found by discussing an auxiliary functional equation

$$
\sum_{k=1}^{n} \lambda_{k}(\varphi(z)) \varphi\left(\alpha^{k} z\right)=F(\varphi(z)) .
$$

Having an analytic solution $\varphi$ of (1.3) invertible in a neighborhood of the origin with $\varphi(0)=0$ we observe that $f(z)=\varphi\left(\alpha \varphi^{-1}(z)\right)$ is the desired solution of (1.2). We took this idea from [7] where the case of constant $\lambda$ 's is considered; cf. also [3, Section 0.1A].

2. Auxiliary equation in case (A). For a real $\delta>0$ we put $U_{\delta}:=$ $\{z \in \mathbb{C}|| z \mid<\delta\}$. By hypotheses in $(\mathrm{H})$ and $(\mathrm{A})$ the function $F$ is invertible in a neighborhood of 0 and $F^{-1}$ is analytic at 0 . Let $G:=F^{-1}$ for simplicity. Then the auxiliary equation (1.3) can be rewritten as

$$
\varphi(z)=G\left(\sum_{k=1}^{n} \lambda_{k}(\varphi(z)) \varphi\left(\alpha^{k} z\right)\right)
$$

in a neighborhood of the origin. Obviously equation (2.4) is of the form

$$
\varphi(z)=h\left(\varphi\left(g_{0}(z)\right), \varphi\left(g_{1}(z)\right), \ldots, \varphi\left(g_{n}(z)\right)\right)
$$

where $g_{k}(z):=\alpha^{k} z$ and

$$
h\left(w_{0}, w_{1}, \ldots, w_{n}\right):=G\left(\sum_{k=1}^{n} \lambda_{k}\left(w_{0}\right) w_{k}\right)
$$

for $z \in U_{\delta}, w_{k} \in U_{\varrho}, k=0, \ldots, n$. Here $\delta, \varrho$ are two small positive constants. Clearly, the function $h: U_{\varrho}^{n+1} \rightarrow \mathbb{C}$ is analytic. Some results of [1], [5] and [9] (see also [3]) on analytic solutions of equation (2.5) will be useful. 
Choose $\alpha=\alpha_{1}$. Since $|\alpha|<1$ as assumed in case (A), let $r$ be the smallest positive integer such that

$$
\left|G^{\prime}(0)\right| \sum_{k=1}^{n}\left|\lambda_{k}(0)\right||\alpha|^{k r}<1 .
$$

Similar to the notations in [3] and [5], let

$$
\begin{aligned}
\left\{w_{k}\right\} & :=\left(w_{0}, w_{1}, \ldots, w_{n}\right), \\
\left\{w_{k, p}\right\} & :=\left(w_{0, p}, w_{1, p}, \ldots, w_{n, p}\right), \quad \forall p \in\{0,1, \ldots, r\} .
\end{aligned}
$$

For each $p \in\{0,1, \ldots, r\}$, define a function $h_{p}: U_{\varrho}^{n+1} \times \mathbb{C}^{p(n+1)} \rightarrow \mathbb{C}$ as follows:

$$
\begin{aligned}
& h_{0}:=h, \quad w_{k, 0}:=w_{k}, \\
& h_{p+1}\left(\left\{w_{k, 0}\right\},\left\{w_{k, 1}\right\}, \ldots,\left\{w_{k, p+1}\right\}\right) \\
& \quad:=\sum_{k=0}^{n} \sum_{q=0}^{p} \frac{\partial h_{p}}{\partial w_{k, q}}\left(\left\{w_{k, 0}\right\},\left\{w_{k, 1}\right\}, \ldots,\left\{w_{k, p}\right\}\right) w_{k, q+1} g_{k}^{\prime}(z) .
\end{aligned}
$$

For the same reasons as for Lemmas 5.6.1 and 5.6.2 in [3], it is easy to prove the following two lemmas by induction, so their proofs are omitted.

Lemma 1. For $p=1, \ldots, r$, the function $h_{p}$ is analytic on $U_{\varrho}^{n+1} \times$ $\mathbb{C}^{p(n+1)}$, and

$$
\begin{aligned}
h_{p}\left(\left\{w_{k, 0}\right\},\left\{w_{k, 1}\right\}, \ldots,\left\{w_{k, p}\right\}\right)= & u_{p}\left(\left\{w_{k, 0}\right\},\left\{w_{k, 1}\right\}, \ldots,\left\{w_{k, p-1}\right\}\right) \\
& +\sum_{k=0}^{n} \frac{\partial h}{\partial w_{k, 0}}\left(\left\{w_{k, 0}\right\}\right) w_{k, p}\left(g_{k}^{\prime}(z)\right)^{p},
\end{aligned}
$$

where $u_{p}: U_{\varrho}^{n+1} \times \mathbb{C}^{(p-1)(n+1)} \rightarrow \mathbb{C}$ is analytic and $u_{1}\left(\left\{w_{k, 0}\right\}\right)=0$.

Lemma 2. Let $\varphi: U_{\delta} \rightarrow U_{\varrho}$ be an analytic function. If

$$
\xi(z):=h\left(\varphi\left(g_{0}(z)\right), \varphi\left(g_{1}(z)\right), \ldots, \varphi\left(g_{n}(z)\right)\right)
$$

on $U_{\delta}$, then the derivatives of $\varphi$ and $\xi$ satisfy

$$
\xi^{(p)}(z)=h_{p}\left(\left\{\varphi\left(g_{k}(z)\right)\right\},\left\{\varphi^{\prime}\left(g_{k}(z)\right)\right\}, \ldots,\left\{\varphi^{(p)}\left(g_{k}(z)\right)\right\}\right), \quad p=1, \ldots, r .
$$

By the definitions of $g_{k}$ and $h$ in (2.6) we have

$$
\begin{aligned}
& \sum_{k=0}^{n} \frac{\partial h}{\partial w_{k, 0}}\left(\left\{w_{k, 0}\right\}\right) w_{k, p}\left(g_{k}^{\prime}(z)\right)^{p} \\
& \quad=\sum_{k=1}^{n} G^{\prime}\left(\sum_{k=1}^{n} \lambda_{k}\left(w_{0,0}\right) w_{k, 0}\right)\left(\lambda_{k}^{\prime}\left(w_{0,0}\right) w_{0, p} w_{k, 0}+\lambda_{k}\left(w_{0,0}\right) w_{k, p} \alpha^{k p}\right)
\end{aligned}
$$


for every $p=1, \ldots, r$. Lemmas 1 and 2 imply that if $\varphi: U_{\delta} \rightarrow U_{\varrho}$ is an analytic solution of equation (2.4) such that $\varphi(0)=0$ then the values

$$
d_{p}:=\varphi^{(p)}(0), \quad p=1, \ldots, r,
$$

satisfy the algebraic system

$$
\left\{\begin{array}{l}
-\frac{P(\alpha)}{F^{\prime}(0)} d_{1}=0 \\
-\frac{P\left(\alpha^{p}\right)}{F^{\prime}(0)} d_{p}=u_{p}\left(\{0\},\left\{d_{1}\right\}, \ldots,\left\{d_{p-1}\right\}\right), \quad p=2, \ldots, r,
\end{array}\right.
$$

where $P$ is defined in our Theorem and $u_{p}$ is given in Lemma 1. Solvability of (2.11) is a necessary condition for the existence of analytic solutions of (2.4).

Lemma 3. Suppose that hypotheses $(\mathrm{H})$ and $(\mathrm{A})$ hold. Then $P\left(\alpha^{p}\right) \neq 0$ for $p=2, \ldots, r$.

Proof. $|\alpha|>0$ since $F^{\prime}(0) \neq 0$. Thus $0<\left|\alpha^{r}\right|<\left|\alpha^{r-1}\right|<\cdots<\left|\alpha^{2}\right|<$ $|\alpha|<1$. By the assumption on $\alpha$ in our Theorem, none of the powers $\alpha^{p}$ $(p=2, \ldots, r)$ is a zero of $P$.

Lemma 3 not only gives solvability of system (2.11) but also implies that the solutions are uniquely determined by $d_{1}$, which can be chosen arbitrarily since $P(\alpha)=0$. Thus we obtain existence of analytic solutions for equation (2.4).

Lemma 4. Suppose that hypotheses (H) and (A) hold. Let $\alpha=\alpha_{1}$ and $r$ be the smallest positive integer satisfying inequality (2.7). For any $\eta_{1} \in \mathbb{C}$, system $(2.11)$ has a unique solution $\left(d_{1}, \ldots, d_{r}\right)$ satisfying $d_{1}=\eta_{1}$ and equation (2.4) has a unique analytic solution $\varphi: U_{\delta} \rightarrow U_{\varrho}$ such that $\varphi(0)=0$, $\varphi^{\prime}(0)=\eta_{1}, \varphi^{(p)}(0)=d_{p}, p=2, \ldots, r$, provided that the radius $\delta$ of the disc $U_{\delta}$ is small enough.

Proof. By Lemma 3 , for any $d_{1}=\eta_{1}$, we can uniquely determine the sequence $\left\{d_{p}\right\}_{p=2}^{r}$ by system (2.11) recursively. We further observe that equation (2.4) is a special case of the functional equation (2.5), which is discussed in [3], and that

$$
\sum_{k=0}^{n}\left|\frac{\partial h}{\partial w_{k, 0}}(\{0\})\left(g_{k}^{\prime}(z)\right)^{r}\right|=\left|G^{\prime}(0)\right| \sum_{k=1}^{n}\left|\lambda_{k}(0)\right||\alpha|^{k r}<1,
$$

which satisfies the condition (7.6.6) in [3] (p. 306). Thus Theorem 7.6.7 in [3] implies that equation (2.4) has a unique analytic solution $\varphi: U_{\delta} \rightarrow U_{\varrho}$ satisfying $\varphi(0)=0, \varphi^{\prime}(0)=\eta_{1}, \varphi^{(p)}(0)=d_{p}, p=2, \ldots, r$, provided that $\delta$ is small enough. 
3. Auxiliary equation in case (B). If hypotheses (H) and (B) hold, choose $\alpha=\alpha_{2}$ in the auxiliary equation (1.3). Clearly $|\alpha|>1$. Let $z=$ $\zeta / \alpha^{n}$ in the auxiliary equation (1.3). This change of variables yields another equivalent equation

$$
\sum_{k=1}^{n} \lambda_{k}\left(\varphi\left(\beta^{n} \zeta\right)\right) \varphi\left(\beta^{n-k} \zeta\right)=F\left(\varphi\left(\beta^{n} \zeta\right)\right)
$$

where $\beta:=1 / \alpha$. Since $\lambda_{n}(z) \neq 0$ in a sufficiently small neighborhood $U_{\varrho}$ of the origin by continuity and by the assumption that $\lambda_{n}(0) \neq 0$ in case (B), we can convert (3.12) into

$$
\varphi(\zeta)=\frac{1}{\lambda_{n}\left(\varphi\left(\beta^{n} \zeta\right)\right)}\left(F\left(\varphi\left(\beta^{n} \zeta\right)\right)-\sum_{k=1}^{n-1} \lambda_{k}\left(\varphi\left(\beta^{n} \zeta\right)\right) \varphi\left(\beta^{n-k} \zeta\right)\right)
$$

in a neighborhood of the origin, which is a functional equation of the form (2.5) with $g_{k}(\zeta):=\beta^{k} \zeta$ and

$$
h\left(w_{0}, w_{1}, \ldots, w_{n}\right):=\frac{1}{\lambda_{n}\left(w_{n}\right)}\left(F\left(w_{n}\right)-\sum_{k=1}^{n-1} \lambda_{k}\left(w_{n}\right) w_{n-k}\right)
$$

for $\zeta \in U_{\delta}, w_{k} \in U_{\varrho}, k=0, \ldots, n$.

Furthermore, one can consider the smallest positive integer $s$ such that

$$
\frac{1}{\left|\lambda_{n}(0)\right|}\left(\left|F^{\prime}(0)\right||\beta|^{n s}+\sum_{k=1}^{n-1}\left|\lambda_{k}(0)\right||\beta|^{(n-k) s}\right)<1
$$

because $|\beta|=1 /|\alpha|<1$. By the definitions of $g_{k}$ and $h$ in (3.14) we have

$$
\begin{aligned}
& \sum_{k=0}^{n} \frac{\partial h}{\partial w_{k, 0}}\left(\left\{w_{k, 0}\right\}\right) w_{k, p}\left(g_{k}^{\prime}(\zeta)\right)^{p} \\
&=\frac{F^{\prime}\left(w_{n, 0}\right) \lambda_{n}\left(w_{n, 0}\right)-F\left(w_{n, 0}\right) \lambda_{n}^{\prime}\left(w_{n, 0}\right)}{\lambda_{n}^{2}\left(w_{n, 0}\right)} w_{n, p} \beta^{n p} \\
&-\sum_{k=1}^{n-1} \frac{\lambda_{k}\left(w_{n, 0}\right)}{\lambda_{n}\left(w_{n, 0}\right)} w_{n-k, p} \beta^{(n-k) p} \\
&-\sum_{k=1}^{n-1} \frac{\lambda_{k}^{\prime}\left(w_{n, 0}\right) \lambda_{n}\left(w_{n, 0}\right)-\lambda_{k}\left(w_{n, 0}\right) \lambda_{n}^{\prime}\left(w_{n, 0}\right)}{\lambda_{n}^{2}\left(w_{n, 0}\right)} w_{n, p} \beta^{n p} w_{n-k, 0}
\end{aligned}
$$

for every $p=1, \ldots, s$. By Lemmas 1 and 2 , if $\varphi: U_{\delta} \rightarrow U_{\varrho}$ is an analytic solution of equation (3.13) such that $\varphi(0)=0$ then the values

$$
d_{p}:=\varphi^{(p)}(0), \quad p=1, \ldots, s,
$$


satisfy the algebraic system

$$
\left\{\begin{array}{l}
Q(\beta) d_{1}=0 \\
Q\left(\beta^{p}\right) d_{p}=u_{p}\left(\{0\},\left\{d_{1}\right\}, \ldots,\left\{d_{p-1}\right\}\right), \quad p=2, \ldots, s,
\end{array}\right.
$$

where $Q(z):=1-\left(F^{\prime}(0) z^{n}-\sum_{k=1}^{n-1} \lambda_{k}(0) z^{n-k}\right) / \lambda_{n}(0)$ in $\mathbb{C}$ and $u_{p}$ is defined as in Lemma 1. Similarly, solvability of the system is a consequence of the following lemma.

Lemma 5. Suppose that hypotheses $(\mathrm{H})$ and $(\mathrm{B})$ hold. Then $Q\left(\beta^{p}\right) \neq 0$ for $p=2, \ldots, s$.

Proof. Note that

$$
Q(z)=\frac{\sum_{k=1}^{n} \lambda_{k}(0) z^{n-k}-F^{\prime}(0) z^{n}}{\lambda_{n}(0)}=\frac{P(1 / z)}{(1 / z)^{n} \lambda_{n}(0)} .
$$

If $\gamma \neq 0$ is a zero of $P(z)$, then $1 / \gamma$ is a zero of $Q(z)$. Since $\alpha=\alpha_{2}$ and $|\alpha|>1$ in case (B), $\beta$ is a zero of $Q(z)$ with the smallest absolute value and $0<|\beta|<1$. Hence $0<\left|\beta^{s}\right|<\left|\beta^{s-1}\right|<\cdots<\left|\beta^{2}\right|<|\beta|<1$. So none of the powers $\beta^{p}(p=2, \ldots, s)$ is a zero of $Q$.

Lemma 5 also implies that solutions of (3.16) are uniquely determined by $d_{1}$, which can be chosen arbitrarily since $Q(\beta)=0$. Thus we obtain existence of analytic solutions for equation (3.13).

LEMMA 6. Suppose that hypotheses $(\mathrm{H})$ and $(\mathrm{B})$ hold. Let $s$ be the smallest positive integer which satisfies inequality (3.15). For any $\eta_{2} \in \mathbb{C}$, system (3.16) has a unique solution $\left(d_{1}, \ldots, d_{s}\right)$ satisfying $d_{1}=\eta_{2}$ and equation (3.13) has a unique analytic solution $\varphi: U_{\delta} \rightarrow U_{\varrho}$ such that $\varphi(0)=$ $0, \varphi^{\prime}(0)=\eta_{2}, \varphi^{(p)}(0)=d_{p}, p=2,3, \ldots, s$, provided that $\delta$ is small enough.

The proof is similar to that of Lemma 4 so we omit it.

\section{Proof of the Theorem and remarks}

Proof of the Theorem. According to Sections 2 and 3 , for any $\eta \in \mathbb{C}$ equation (1.3) has a unique analytic solution $\varphi$ in a neighborhood of the origin satisfying $\varphi(0)=0$ and $\varphi^{\prime}(0)=\eta$. For $\eta \neq 0$ the inverse $\varphi^{-1}(z)$ is well defined and analytic in a neighborhood of the origin. Let

$$
f(z)=\varphi\left(\alpha \varphi^{-1}(z)\right) .
$$

Obviously, $f$ is also analytic in a neighborhood of the origin and $f^{k}(z)=$ $\varphi\left(\alpha^{k} \varphi^{-1}(z)\right)$ for any integer $k>0$. Hence,

$$
\sum_{k=1}^{n} \lambda_{k}(z) f^{k}(z)=\sum_{k=1}^{n} \lambda_{k}\left(\varphi\left(\varphi^{-1}(z)\right)\right) \varphi\left(\alpha^{k} \varphi^{-1}(z)\right)=F\left(\varphi\left(\varphi^{-1}(z)\right)\right)=F(z) .
$$


That is, $f$ satisfies equation (1.2) in a neighborhood of the origin. Furthermore, $f(0)=0$ and $f^{\prime}(0)=\alpha$. The proof of the Theorem is complete.

Consider a concrete iterative equation

$$
\left(\frac{1}{2}+\sin z\right)^{2} f(z)-4 \cos ^{5} z f^{2}(z)+(\cos (2 z)+\sin z)^{3} f^{3}(z)=z e^{z}
$$

It has the form (1.2) with $\lambda_{1}(z)=(1 / 2+\sin z)^{2}, \lambda_{2}(z)=-4 \cos ^{5} z, \lambda_{3}(z)=$ $(\cos (2 z)+\sin z)^{3}$ and $F(z)=z e^{z}$. Obviously, the functions $\lambda_{1}, \lambda_{2}, \lambda_{3}$ and $F$ are analytic, $F(0)=0, F^{\prime}(0)=1$ and $\lambda_{3}(0)=1$. Moreover, the polynomial

$$
\lambda_{1}(0) z+\lambda_{2}(0) z^{2}+\lambda_{3}(0) z^{3}-F^{\prime}(0)
$$

has exactly three complex zeros $\pm i / 2$ and 4 . Since two of them are less than 1 and the other is greater than 1 in absolute value, our Theorem guarantees that equation (4.18) has at least three analytic solutions in a neighborhood of the origin.

Existence of analytic solutions remains unsettled in the remaining cases:

(i) all zeros of $P(z)$ are 1 in absolute value,

(ii) all zeros are not greater than 1 in absolute value and at least one zero is less than 1 in absolute value, but $F^{\prime}(0)=0$,

(iii) all zeros are not less than 1 in absolute value and at least one is greater than 1 but the leading coefficient $\lambda_{n}(z)$ is a nonzero function with $\lambda_{n}(0)=0$.

Acknowledgements. The author is grateful to Prof. Weinian Zhang for his advice and to Prof. Janusz Matkowski (University of Zielona Góra, Poland) for helpful discussions and encouragement when he visited Sichuan University. The author also thanks Prof. Jianguo Si for his suggestion.

\section{References}

[1] K. Baron, R. Ger and J. Matkowski, Analytic solutions of a system of functional equations, Publ. Math. Debrecen 22 (1975), 189-194.

[2] K. Baron and W. Jarczyk, Recent results on functional equations in a single variable, perspectives and open problems, Aequationes Math. 61 (2001), 1-48.

[3] M. Kuczma, B. Choczewski and R. Ger, Iterative Functional Equations, Encyclopedia Math. Appl. 32, Cambridge Univ. Press, Cambridge, 1990.

[4] J. H. Mai and X. H. Liu, Existence, uniqueness and stability of $C^{m}$ solutions for a kind of iterative functional equation, Sci. in China A30 (2000), 129-144 (in Chinese).

[5] J. Matkowski, On the uniqueness of differentiable solutions of a functional equation, Bull. Acad. Polon. Sci. Sér. Sci. Math. Astronom. Phys. 18 (1970), 253-255.

[6] J. Si, Existence of local analytic solutions of of the iterative equation $\sum_{i=1}^{n} \lambda_{i} f^{i}(z)=$ $F(z)$, Acta Math. Sinica 37 (1994), 590-600 (in Chinese).

[7] J. Si and X. Wang, Analytic solution of a polynomial-like iterate functional equation, Demonstratio Math. 32 (1999), 95-103. 
B. $\mathrm{Xu}$

[8] J. Si and X. Wang, Differentiable solutions of a polynomial-like iterative equation with variable coefficients, Publ. Math. Debrecen 58 (2001), 57-72.

[9] W. Smajdor, Local analytic solutions of the functional equation $\Phi(z)=H\left(z, \Phi\left[f_{1}(z)\right]\right.$, $\left.\ldots, \Phi\left[f_{n}(z)\right]\right)$, Ann. Polon. Math. 24 (1970), 39-43.

[10] J. Zhang, L. Yang and W. Zhang, Some advances on functional equations, Adv. Math. (China) 24 (1995), 385-405.

[11] W. Zhang, Discussion on the iterated equation $\sum_{i=1}^{n} \lambda_{i} f^{i}(x)=F(x)$, Chin. Sci. Bull. 32 (1987), 1444-1451.

[12] - Stability of the solution of the iterated equation $\sum_{i=1}^{n} \lambda_{i} f^{i}(x)=F(x)$, Acta Math. Sci. 8 (1988), 421-424.

[13] - Discussion on the differentiable solutions of the iterated equation $\sum_{i=1}^{n} \lambda_{i} f^{i}(x)$ $=F(x)$, Nonlinear Anal. 15 (1990), 387-398.

[14] - Solutions of equivariance for a polynomial-like iterative equation, Proc. Roy. Soc. Edinburgh Sect. A 130 (2000), 1153-1163.

[15] W. Zhang and J. A. Baker, Continuous solutions of a polynomial-like iterative equation with variable coefficients, Ann. Polon. Math. 73 (2000), 29-36.

Department of Mathematics

Sichuan University

Chengdu, Sichuan 610064, P.R. China

E-mail: xb4106@sina.com

xb0408@sohu.com

Reçu par la Rédaction le 28.8.2002

Révisé le 1.12.2003 et 4.3.2004 